\title{
Bienestar subjetivo y psicológico: comparación de jóvenes y adultos mayores
}

\section{Subjective and psychological well-being: young and older adults' comparison}

\author{
Juan C. Meléndez ${ }^{1}$, Ana I. Agustí ${ }^{1}$, Iraida Delhom¹, María Fernanda Reyes Rodríguez ${ }^{2}$, Encarnación Satorres ${ }^{1}$. \\ 1Universidad de Valencia, España \\ 2Universidad El Bosque, Colombia. \\ (Rec.: julio de 2017 - octubre de 2017)
}

\section{Resumen}

El bienestar es un constructo complejo que se refiere a la experiencia óptima y el funcionamiento. El bienestar subjetivo, basado en la perspectiva hedónica, es la valoración general de la vida y el bienestar psicológico, fundamentado en la perspectiva eudaimónica, se asocia al desarrollo personal y realización del potencial de uno mismo. Este trabajo compara un grupo de jóvenes con un grupo de adultos mayores en medidas de bienestar subjetivo y del bienestar psicológico, para estudiar los posibles cambios asociados a la edad. Se realizó un estudio correlacional, en el que participaron 148 sujetos pertenecientes a dos grupos de edad: jóvenes y adultos mayores de Valencia (España). Los resultados mostraron diferencias en el bienestar subjetivo con puntuaciones más altas en el grupo de los adultos mayores para las escalas de afecto positivo. Para el bienestar psicológico los adultos mayores obtuvieron puntuaciones significativamente mayores en las dimensiones de autoaceptación, autonomía y dominio del ambiente. Los resultados apoyan las teorías sobre el sesgo de positividad en la vejez y un mayor uso de estrategias de tipo acomodativo como forma de adaptación a las nuevas situaciones.

Palabras clave: jóvenes, adultos mayores, bienestar subjetivo; bienestar psicológico.

\begin{abstract}
Well-being is a complex construct that refers to optimal experience and functioning. Subjective well-being, based on the hedonic perspective, refers to general assessment of life, and psychological well-being, based on the eudemonic perspective, is associated with personal development and fulfillment of one's potential. This study compares a group of young people with a group of elderly people by means of subjective well-being and psychological well-being, in order to study the possible changes associated with age. A correlational study was carried out in which there were 148 subjects from two age groups: young people and elderly people from Valencia (Spain). The results revealed differences in subjective well-being, with higher scores in the group of elderly people for the scales of positive affect. While in psychological well-being, elderly people got significantly higher scores in the dimensions of self-acceptance, autonomy and environmental mastery. These results support theories of positivity bias in old age, and a greater use of accommodative strategies as means of adaptation to new situations.
\end{abstract}

Keywords: young people, elderly people, subjective well-being; psychological well-being.

Correspondencia: Juan C. Meléndez. Departamento de Psicología Evolutiva y de la Educación, Facultad de Psicología, Universidad de Valencia (España). Av. Blasco Ibáñez 21, Valencia, ES 46010; Tfno: +34963983844.

correo electrónico: melendez@uv.es 


\section{Introducción}

La psicología ha prestado una importante atención a los aspectos patológicos del ser humano descuidando al individuo satisfecho y las condiciones que pueden facilitar un desarrollo benigno basado en la mejora personal. Seligman y Csikszentmihalyi (2000) planteaban que el objetivo de la psicología positiva debía ser catalizar un cambio en el enfoque de la psicología que fuera desde la preocupación por la reparación de las peores cosas en la vida a la construcción de cualidades positivas. Uno de los conceptos clave de esta perspectiva que ha sido investigado ampliamente en los últimos años es el bienestar.

El bienestar es una construcción compleja que se refiere a la experiencia óptima y el funcionamiento (Deci \& Ryan, 2008). En la investigación psicológica y médica coexisten dos perspectivas generales sobre el bienestar: el enfoque hedónico, que se centra en la felicidad y define el bienestar en términos de logro y de evitación del dolor; y el enfoque eudaimónico, que se centra en el significado y la autorrealización y define el bienestar en términos del grado en que una persona está funcionando plenamente (Ryan \& Deci, 2001). Existe una creciente conciencia entre los investigadores de que el bienestar no es la ausencia de enfermedad mental (Cacioppo \& Berntson, 1999), sino que implica un desarrollo positivo del estado físico, mental y social.

El bienestar subjetivo, basado en la perspectiva hedónica, es cercano a conceptos de larga tradición en la psicología y, actualmente, engloba términos como felicidad, moral o satisfacción vital. Veenhoven (1994) definió el bienestar subjetivo como el grado en que una persona juzga de un modo general o global su vida utilizando dos componentes: uno de carácter emocional y otro cognitivo. Para Diener y Lucas (1999), el componente emocional o afectivo, está relacionado con los sentimientos de placer y displacer que experimenta la persona (felicidad) y el segundo componente de carácter más cognitivo, está relacionado con el juicio que merece a la persona su trayectoria evolutiva (satisfacción).

La perspectiva eudaimónica, centrada en el bienestar psicológico, se refiere al desarrollo personal como una función de las habilidades individuales, la realización del potencial de uno mismo. El bienestar psicológico se define, según Ryff y Keyes (1995), como un esfuerzo para perfeccionarse y cumplir con nuestro potencial, lo cual tiene que ver con tener un propósito en la vida, con que la vida adquiera significado para uno mismo, con los desafíos y con un cierto esfuerzo por superarlos y conseguir metas valiosas (Ryff, 2002; Ryff \& Keyes, 1995). A partir de los trabajos de Ryff (1989), se definen criterios y cualidades concretas y estables fundamentados en diferentes tipos de precedentes tanto médicos como psicológicos, que abogan por la definición positiva de la salud más allá de la ausencia de enfermedad. Se proponen seis dimensiones a partir de las cuales se desarrollaron las Escalas de Bienestar Psicológico (Ryff, 1989). Estas seis dimensiones son: autonomía (capacidad de mantener la independencia y la individualidad en diferentes contextos y para resistir la presión social); dominio del ambiente (capacidad de elegir o crear entornos favorables para satisfacer metas y necesidades); autoaceptación (tener una percepción exacta de las propias acciones, motivos y sentimientos); relaciones positivas con los demás (confianza mutua y empatía); crecimiento personal (dedicación a la realización del potencial personal para seguir creciendo); y propósito en la vida (establecer metas y definir objetivos para dar dirección a la vida). Keyes, Shmotkin y Ryff (2002) indican que el bienestar subjetivo y el psicológico están relacionados, y aunque ambos poseen dimensiones que no comparten con el otro, existe un cierto solapamiento entre ellos en las dimensiones de autoaceptación y dominio del ambiente, a la par que el bienestar psicológico mantiene dimensiones que son exclusivas: propósito en la vida y crecimiento personal.

Aunque los cambios objetivos y subjetivos que suceden a medida que nos hacemos mayores podrían hacernos pensar que nuestro bienestar subjetivo se vería afectado, la investigación señala que este aspecto muestra una marcada estabilidad con el paso de los años, siendo los niveles alcanzados por las personas mayores similares a los que muestran personas en décadas anteriores de la vida. De esta forma, el bienestar subjetivo como medida global parece no experimentar cambios significativos asociados a la edad, ni en estudios de tipo transversal ni en estudios longitudinales (Morganti, Nehrke, Hulicka \& Cataldo, 1988; Mroczek \& Kolarz, 1998; Okun \& Stock, 1987). En cuanto a los cambios del bienestar psicológico según aumenta la edad, diversos estudios han informado que algunas de sus dimensiones exhiben claramente los efectos de edad, mientras que otras se mantienen estables (Meléndez, Tomás, \& Navarro, 2008, 2011; Ryff, 1991). Específicamente, las dimensiones relaciones positivas con los demás y autoaceptación han demostrado ser relativamente estables. La dimensión dominio del ambiente, por su parte, tiende a ser más elevada en adultos mayores y de mediana edad que en los jóvenes, pero se mantiene estable desde la edad media hasta la vejez (Keyes et al., 2002). La dimensión autonomía exhibe un patrón similar, aunque menos marcado. Por último, las dos dimensiones restantes, propósito en la vida y crecimiento personal, consideradas las más características del bienestar psicológico, disminuyen según se avanza en el desarrollo (Ryff, 2014). Esta disminución es especialmente fuerte cuando se comparan adultos de mediana edad y adultos mayores.

Este trabajo pretende comparar un grupo de jóvenes con un grupo de adultos mayores en medidas de bienestar subjetivo y en las dimensiones del bienestar psicológico, para estudiar los posibles cambios asociados a la edad. Así, se espera que, dada la estabilidad del bienestar subjetivo, no se observen diferencias entre los grupos, mientras que en el bienestar psicológico la dimensión dominio del ambiente debería mostrar un aumento con la edad, y las dimensiones de crecimiento personal y propósito en la vida deberían descender.

\section{Método}

\section{Participantes}

El diseño de investigación es correlacional y tipo encuesta. La selección de la muestra se realizó a través de un muestreo intencional y la componen un total de 148 sujetos pertenecientes a dos grupos de edad: jóvenes $(N=75$; edad media $=20.62$ años, $\mathrm{DE}=2.51$, rango 18-30 años) y adultos mayores $(\mathrm{N}=73$; edad media $=79.56$ años, $\mathrm{DE}=8.21$, rango $65-90$ años), quienes asistían a centros de jubilados de la ciudad de Valencia (España).

Como criterios para la diferenciación de los grupos de edad, el grupo de jóvenes se inicia a partir de los 18 años, por marcar el punto legal de entrada en la mayoría de edad. En el caso de los 
adultos mayores, se tomaron edades iguales o superiores a 65 años por ser la edad normativa de jubilación. Además, como criterios de exclusión se tuvo en cuenta que no estuvieran institucionalizados y no existiera deterioro cognitivo, siendo excluidos aquellos participantes con una puntuación inferior a 24 en el Mini Examen Cognitivo (Lobo, Saz \& Marcos, 2002). En relación a sus características sociodemográficas, el grupo de jóvenes estuvo compuesto por un $73,3 \%$ de mujeres, un $84,7 \%$ estaba soltero y un $15,3 \%$ vivía en pareja. Un $16,1 \%$ tenía finalizados estudios secundarios y un 83,9\% tenía estudios universitarios. El grupo de adultos mayores estuvo compuesto por un $68,5 \%$ de mujeres, un $69,8 \%$ de integrantes casados, un $6,8 \%$ solteros y un $23,3 \%$ viudos. Los niveles académicos fueron de un $19,2 \%$ de los integrantes del grupo con estudios incompletos de primaria, un $41,1 \%$ con estudios de primaria completos, un $17,8 \%$ con secundaria finalizada y un $21,9 \%$ que poseía estudios universitarios.

\section{Instrumentos}

Escalas de Afecto Positivo y Negativo (PANAS). Fueron desarrolladas por Watson, Clark y Tellegen (1988) para evaluar las dimensiones del afecto a través de dos factores, uno que evalúa el afecto positivo (AP) y otro, el afecto negativo (AN). El AP se refiere al nivel en que las personas experimentan emociones placenteras, mientras que AN es una dimensión general relativa a la experimentación de estados de ánimo aversivos. El cuestionario incluye 20 ítems, 10 de los cuales se refieren a la sub-escala de afecto positivo (AP) y 10 a la sub-escala de afecto negativo (AN). Las personas deben puntuar en una escala Likert de cinco niveles ( $1=$ muy poco o nada, $2=$ algo, $3=$ moderadamente, $4=$ bastante, $5=$ extremadamente) el grado en que han experimentado estos descriptores del afecto en la última semana y generalmente. Nolla, Queral y Miró (2014) señalan que esta diferenciación puede ser utilizada para valorar el afecto tanto en su forma de estado (en este caso se pide al sujeto que informe en relación a la última semana -incluyendo el día cuándo se informa) como de rasgo (quien informa debe reflejar su respuesta considerando su estado usual). El estudio de Sandín et al. (1999) evidencia con un gran nivel de consistencia la aparición de los dos factores que, aunque correlacionan de forma negativa (muy baja) y significativa, aparecen como dimensiones independientes, no como dos aspectos opuestos de un mismo fenómeno.

Finalmente, se aplicaron las escalas de bienestar psicológico de Ryff (1989), que se han adaptado al contexto español (Meléndez et al., 2008). Estas escalas conceptualizan y miden el bienestar con seis dimensiones: autoaceptación (tener una percepción exacta de sus acciones, motivos y sentimientos), relaciones positivas con los demás (confianza y empatía mutuas), autonomía (capacidad de mantener la independencia y la individualidad en diferentes contextos y resistencia a la presión social), dominio del ambiente (capacidad de elegir o crear ambientes propicios para alcanzar metas y necesidades), propósito en la vida (fijar objetivos y definir objetivos para dar dirección a la vida) y crecimiento personal (dedicación a la realización de potencial personal para seguir creciendo). Los ítems son evaluados desde 1 (totalmente en desacuerdo conmigo) a 6 (totalmente de acuerdo conmigo).

\section{Procedimiento}

La muestra fue obtenida en centros de jubilados de la ciudad de Valencia (España) en los que se pidió la participación voluntaria tanto de los adultos mayores como de jóvenes que bien acompañaban a los anteriores bien asistían ellos mismos a los centros a realizar alguna actividad. Las personas interesadas firmaron un consentimiento informado en el que se estipulaba el carácter voluntario, confidencial y anónimo de su participación en la investigación. Posteriormente, se aplicaron las escalas y se realizó el análisis de datos.

\section{Análisis de datos}

Se realizó análisis multivariado a través de MANOVAs para determinar si había diferencias entre los grupos de jóvenes y adultos mayores en las medidas de bienestar subjetivo y en las dimensiones del bienestar psicológico. A continuación, se realizaron pruebas t para analizar las diferencias entre los grupos en las variables. Los datos fueron analizados con SPSS 21.

\section{Resultados}

En primer lugar, se realizó un MANOVA para comparar las puntuaciones de los grupos en las medidas de bienestar subjetivo. Los contrastes multivariados mostraron un efecto principal de grupo $(\Lambda=.225, \mathrm{~F}[4,141]=9.67, p<0.001, \mathrm{~h} 2=.225)$. Dado que se hallaron diferencias significativas se analizó cada variable dependiente. Se encontraron diferencias significativas en afecto positivo de última semana $(F[1,146]=9.38, p=$ $\left.0.003, h^{2}=.061\right)$ donde los adultos mayores obtuvieron puntuaciones medias (35.64) más altas que los jóvenes (31.94), y para afecto positivo generalmente $(F[1,146]=29.03, p<$ $\left.0.001, h^{2}=168\right)$ con medias más altas en los adultos mayores (38.63) que en los jóvenes (32.28).

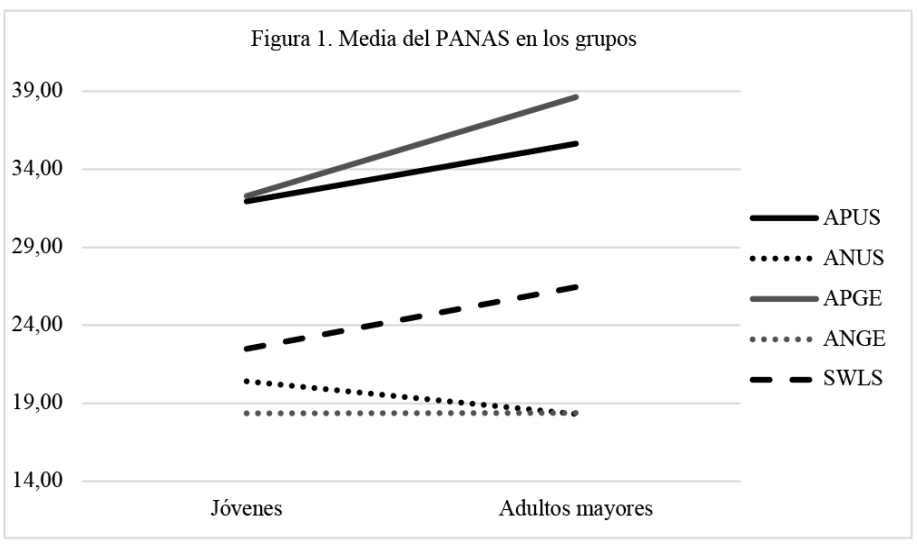

Figura 1. Medias del PANAS en los grupos. Se registra APUS = Afecto positivo última semana; ANUS = Afecto negativo última semana; $\mathrm{APGE}=$ Afecto positivo generalmente; $\mathrm{ANGE}=$ Afecto negativo generalmente.

A continuación, se puso a prueba un MANOVA para comparar los grupos en las dimensiones de bienestar psicológico. Los contrastes multivariados señalaron un efecto principal de grupo $\left(\Lambda=.348, F[6,141]=12.54, p<0.001, h^{2}=.348\right)$. Al estudiar la existencia de diferencias sobre cada variable dependiente se encontraron diferencias en todas las dimensiones excepto 
en propósito en la vida; autoaceptación $(F[1,148]=34.49, p$ $\left.<0.001, \mathrm{~h}^{2}=.192\right)$, relaciones positivas $(\mathrm{F}[1,148]=9.54, p=$ $\left.0.002, h^{2}=.061\right)$, autonomía $\left(F[1,148]=10.31, p=.002, h^{2}=\right.$ $.066)$, dominio del ambiente $\left(F[1,148]=6.79, p=0.010, h^{2}\right.$ $=.044)$, crecimiento personal $(\mathrm{F}[1,148]=8.95, p=0.003, \mathrm{~h} 2$ $=.058)$, siendo las medias de los adultos mayores más altas excepto en las dimensiones de relaciones positivas con otros y crecimiento personal.

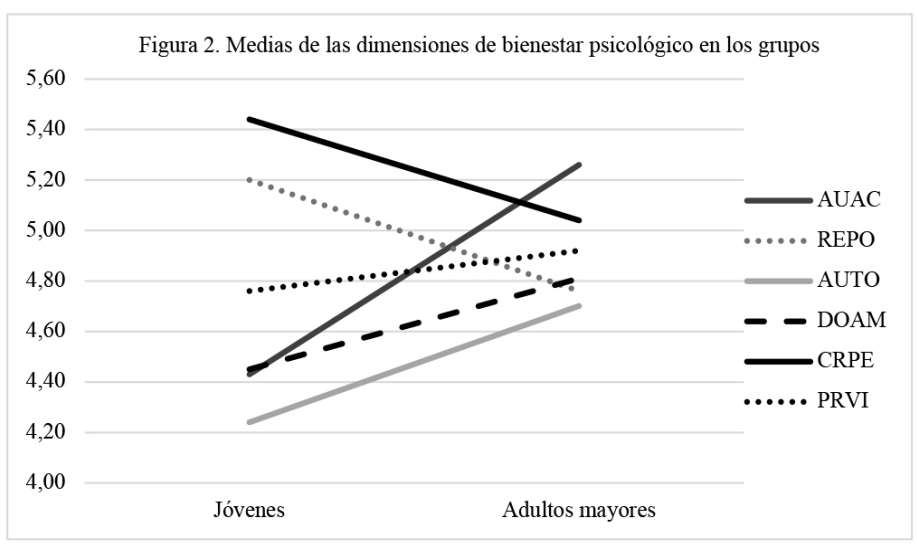

Figura 2. Medias de las dimensiones de bienestar psicológico en los grupos. Se registra $\mathrm{AUAC}=$ autoaceptación; $\mathrm{REPO}=$ relaciones positivas con otros; $\mathrm{AUTO}=$ autonomía; $\mathrm{DOAM}=$ Dominio del ambiente; $\mathrm{CRPE}=$ Crecimiento personal; $\mathrm{PRVI}=$ Propósito en la vida.

\section{Discusión}

Este trabajo compara dos grupos, jóvenes y adultos mayores, en medidas de bienestar subjetivo y psicológico. Dada la estabilidad evolutiva del bienestar subjetivo no se esperaba observar diferencias entre los grupos, mientras que sí se esperaban encontrar menores puntuaciones en el grupo de adultos mayores en las dimensiones de crecimiento personal y propósito en la vida del bienestar psicológico. Tal y como se ha observado en los resultados, las hipótesis se han cumplido parcialmente. En relación al bienestar subjetivo, se han obtenido diferencias en el afecto positivo con puntuaciones más altas en los adultos mayores. En cuanto al bienestar psicológico, los adultos mayores obtuvieron medias más altas en las dimensiones de autoaceptación, autonomía y dominio del ambiente. En las dimensiones de relaciones positivas con otros y crecimiento personal, son los jóvenes los que presentan medias significativamente más altas.

El bienestar subjetivo se relaciona con los sentimientos de placer y displacer (afectos positivos y afectos negativos) que experimenta la persona, y, por tanto, se refiere a lo que las personas piensan y sienten acerca de sus vidas, y cómo evalúan su existencia (Diener, 2012). Las diferencias obtenidas señalan que los adultos mayores muestran significativamente mayor cantidad de afectos positivos y satisfacción que los jóvenes. Desde la Teoría de la Selectividad Socioemocional (TSS) de Carstensen (1992) se ofrecen diversas explicaciones que afianzan estos resultados. En este sentido Carstensen, Pasupathi, Mayr y Nesselroade (2000) señalaron que después de los 60 años la frecuencia de emociones negativas disminuye y aumentan los periodos de experiencias emocionales positi- vas. En la vejez las personas seleccionan situaciones que les exigen una menor respuesta emocional negativa; además, como perciben que el tiempo futuro es más corto, priorizan las metas que significan una experiencia emocional positiva (Sims, Hogan \& Carstensen, 2015). De este modo, si a medida que las personas se hacen mayores se autorregulan emocionalmente de forma más eficiente, es comprensible que cambien también aquello a lo que prestan atención.

Esta cuestión fue analizada por Mather y Carstensen (2003) a partir de dos experimentos. El primero fue una tarea dot-probe en el que tras la aparición del punto de fijación central aparecían las versiones neutra y emocional de una cara, desaparecían y aparecía un punto gris en una de las posiciones donde estaban las fotografías teniendo el participante que presionar la tecla correspondiente del teclado; el segundo experimento de reconocimiento se realizó mediante una tarea de elección forzada en la que a los participantes se les mostraron pares de rostros combinados por su expresión emocional y se les preguntaba qué rostro habían visto antes; mediante estos experimentos se comprobó la existencia de sesgos atencionales a partir de la evitación de caras negativas y de una mayor atención a caras que expresan emociones positivas. Los resultados que obtuvieron indican que los adultos mayores parecen ser más lentos en identificar un estímulo objetivo cuando este aparece tras una cara negativa y en cambio, parecen responder más rápidamente cuando el target aparece tras una cara positiva. Además, los adultos mayores muestran una mejor ejecución para aquellos rostros cuya expresión es positiva en comparación con las de valencia negativa. Mather y Carstensen (2003) ponen el énfasis en la necesidad de ser conscientes de cómo el sesgo atencional muestra resultados a favor de los estímulos de carácter positivo, de manera que los participantes se focalizan en mayor medida en objetivos y metas con un alto poder emocional, consiguiendo distraer o alejar su atención de aquella información que, emocionalmente, no les hace ningún bien.

Por otra parte, desde los estudios cognitivos centrados en la memoria autobiográfica con relación a la valencia emocional del recuerdo, Gallo, Korthauer, McDonough, Teshale y Johnson (2011) señalaron que los adultos mayores tienden a recordar los acontecimientos de sus vidas de forma más positiva que los adultos jóvenes. De este modo, el número de eventos positivos en relación con los acontecimientos negativos tiende a aumentar con la edad, produciéndose un sesgo atencional de positividad. Este sesgo de positividad en la memoria autobiográfica parece ser un factor adaptativo que contribuye a mejorar el estado de ánimo y aumentar las emociones positivas en las personas mayores (Pasupathi \& Carstensen, 2003), de manera que recordar las cosas de una forma positiva podría ser una estrategia efectiva de regulación emocional. De modo similar, Comblain, D'Argembeau y Van der Linden (2005) demostraron que cuando se les pedía a sujetos adultos que evocaran recuerdos positivos y negativos, y proporcionaran detalles al respecto, los sujetos más mayores evocaban más recuerdos positivos y mostraban además una mayor intensidad emocional, comparados con los adultos más jóvenes. Así pues, los adultos mayores deberían estar especialmente motivados para procesar lo positivo de sus experiencias personales sobre los detalles negativos. Ello con el fin de regular sus emociones (Schryer \& Ross, 2014) y así potenciar la protección de su bienestar emocional actual mediante el recuerdo autobiográfico positivo. 
Respecto al bienestar psicológico, se ha comprobado que los jóvenes obtienen puntuaciones más altas que los adultos mayores en la dimensión de relaciones positivas con otros, resultados similares a otros trabajos que muestran una disminución según aumentaba la edad. Parece ser que, desde la mediana edad, existe una tendencia a seleccionar los contactos sociales. No obstante, esta tendencia es muy selectiva y afecta sobre todo a los contactos menos cercanos; las relaciones más estrechas y proveedoras de apoyo permanecen básicamente intactas con la edad (Meléndez, Tomás \& Navarro, 2007). Según Brandtstädter y Renner (1990), la reducción en los contactos sociales y la progresiva desaparición de las relaciones íntimas es asumida por las personas mayores, ya que tienden a aplicar estrategias de tipo acomodativo como forma de adaptación a las nuevas situaciones.

Por otra parte, la aplicación de este tipo de estrategias puede explicar la reducción del crecimiento personal con la edad, ya que su puesta en marcha genera un ajuste de las preferencias y de las metas que, aun no siendo intencional, hace que el sujeto se adapte adecuadamente a la nueva situación, disminuyendo la percepción de las restricciones situacionales o unos recursos personales insuficientes para conseguir metas antes valoradas. Tomamos como premisa de partida que el envejecimiento implica una reducción de los recursos personales de acción y de las opciones evolutivas disponibles, lo cual tiende a dar protagonismo a la acomodación frente a la asimilación como estrategia de adaptación y optimización de los recursos. También desde la TSS, se ofrece una posible explicación a estas diferencias en la dimensión crecimiento personal. La TSS postula que los adultos jóvenes perciben el tiempo de forma expansiva, más abierto, por tanto, es más probable que las metas sean de preparación, recopilación de información, experimentación de la novedad y expansión de amplitud de conocimientos con el fin de prepararse para eventos futuros. En cambio, los adultos mayores perciben el tiempo de modo limitado, y los objetivos se centran más en la propia regulación emocional para optimizar el bienestar y dar significado a la vida (Fredrickson \& Cartensen, 1990; Fung, Carstensen \& Lutz, 1999).

Por contra, tres dimensiones altamente relacionadas con salud mental positiva, el self y su estabilidad (Jahoda, 1958) muestran puntaciones significativamente más altas en el grupo de adultos mayores: autoaceptación (reconocer y aceptar múltiples aspectos de sí mismo, incluyendo buenas y malas cualidades), autonomía (capacidad de resistir las presiones sociales para pensar y actuar de ciertas maneras, evaluándose por los estándares personales) y dominio del ambiente (capacidad de elegir o crear contextos adecuados a las necesidades y valores personales).

Aunque en trabajos anteriores (Keyes et al., 2002; Ryff, 1989) la autoaceptación se ha mostrado como una dimensión relativamente estable, debe tenerse en cuenta que para Allport (1961) una adecuada autoaceptación es la base del funcionamiento óptimo y un rasgo de madurez lo que podría explicar las diferencias obtenidas. En esta misma línea y desde las teorías del ciclo vital, también se enfatiza la importancia de esta dimensión para la obtención de la integridad planteada por Erikson (1959). Así pues, en la juventud, una de las tareas principales es la consecución de la intimidad basada en la capacidad para comprometerse a afiliaciones y grupos concretos, y la lealtad a los principios éticos derivados de los compromisos. En este sentido, la consecución de la integridad implica la aceptación del propio y único ciclo de vida como algo que debiese ser y que, necesariamente, no permite sustitución alguna. Además, y en relación a los resultados del bienestar subjetivo, desde un enfoque cuantitativo, Ryff y Keyes (1995) señalan que la autoaceptación y el dominio del ambiente están relacionados con medidas de felicidad y satisfacción con la vida, por tanto y con base en los resultados obtenidos y el sesgo de positividad señalado, sería coherente esperar puntuaciones significativamente más altas en los adultos mayores.

Por lo que respecta a la autonomía, se han obtenido resultados contradictorios; según Ryff (1989) o Ryff y Keyes (1995), existen diferencias entre jóvenes y adultos de mediana edad, pero no entre jóvenes y adultos mayores. En cambio, otros trabajos señalan un aumento significativo de esta dimensión con la edad (Mayordomo, Sales, Satorres \& Meléndez, 2016). Posiblemente, las diferencias obtenidas son debidas a que la juventud, como inicio de la vida adulta, supone una importante oportunidad de crecimiento, pero a su vez, puede suponer un período de considerable estrés e incertidumbre, producto de los continuos cambios y de los nuevos desafíos. A diferencia de los adultos mayores, a los jóvenes todavía les es difícil sostener su propia individualidad en diversos contextos sociales, tomando como base sus propias convicciones y tratando de mantener su independencia. Esto es debido a la mayor necesidad de aprobación de los demás en la construcción de su intimidad. También Neugarten (1973) señala la importancia de volver la mirada hacia dentro en los últimos años de la vida, logrando una sensación de libertad de las normas que nos rigen en nuestro desarrollo continuado.

Finalmente, los resultados obtenidos en dominio del ambiente son coherentes con trabajos anteriores (Ryff, 1989; Ryff \& Keyes, 1995) en los que se destaca un incremento significativo de esta dimensión con la edad. El envejecimiento sitúa a las personas en un contexto caracterizado por cambios que suponen una situación desventajosa y sobre los que no existe un control interno para su modificación. Por tanto, existe una clara necesidad de adaptación y ajuste a la nueva realidad. Dichos cambios pueden verse atenuados por el proceso de adaptación mediante el dominio de los entornos de desarrollo, entendido como un proceso que se centra en la eficacia de la persona para cambiar el funcionamiento, de manera que se logre un envejecimiento exitoso. Este aspecto adquiere una gran importancia en la última etapa del ciclo vital en la que el énfasis en crear o hallar un contexto inmediato que favorezca las capacidades y necesidades personales es altamente adaptativo.

Por último, señalar como principales limitaciones de este estudio el muestreo no probabilístico y su diseño de investigación que futuras investigaciones deberían intentar superar, obteniendo datos de carácter longitudinal, lo que facilitaría la generalización de los resultados. 


\section{Referencias}

Allport, G. (1961). Pattern and growth in personality. New York: Holt, Rinehart \& Winston.

Brandtstädter, J. \& Renner, G. (1990). Tenacious goal pursuit and flexible goal adjustment: explication and age-related analysis of assimilative and accommodative strategies of coping. Psychology \& Aging, 5, 58-67. doi:10.1037/0882-7974.5.1.58

Cacioppo, J. \& Berntson, G. (1999). The affect system: Architecture and operating characteristics. Current Directions in Psychological Science, 8, 133-137. doi:10.1111/1467-8721.00031

Carstensen, L. (1992). Social and emotional patterns in adulthood: Support for socioemotional selectivity theory. Psychology \& Aging, 7(3), 331-338. doi:10.1037/0882-7974.7.3.331

Carstensen, L., Pasupathi, M., Mayr, U. \& Nesselroade, J. (2000). Emotional experience in everyday life across the adult life span. Journal of Personality and Social Psychology, 79(4), 644-655. doi:10.1037/00223514.79.4.644

Comblain, C., D'Argembeau, A. \& Van der Linden, M. (2005). Phenomenal characteristics of autobiographical memories for emotional and neutral events in older and younger adults. Experimental Aging Research, 31(2), 173-189. doi:10.1080/03610730590915010

Deci, E. \& Ryan, R. (2008). Hedonia, eudaimonia, and well-being: An introduction. Journal of Happiness Studies, 9, 1-11. doi:10.1007/s10902-006-9018-1

Diener, E. (2012). New findings and future directions for subjective well-being research. American Psychologist, 67, 590-597. doi:10.1037/a0029541

Diener, E. \& Lucas, R. (1999). Personality and subjective weIl-being. En D. Kahneman, E. Diener \& N. Schwarz (Eds.), Well-being: The foundations of hedonic psychology (pp. 213-227). New York: Russell Sage.

Erikson, E. H. (1959). Identity and the life cycle: Selected papers. Psychological Issues, 1, 1-171. Recuperado en: http://psycnet.apa.org/record/1960-02756-001

Fredrickson, B. \& Carstensen, L. (1990). Choosing social partners: How old age and anticipated endings make people more selective. Psychology \& Aging, 5, 335347. doi:10.1037/0882-7974.5.3.335

Fung, H., Carstensen, L. \& Lutz, A. (1999). Influence of time on social preferences: Implications for life-span development. Psychology \& Aging, 14(4), 595-604. doi:10.1037/0882-7974.14.4.595

Gallo, D., Korthauer, L., McDonough, I., Teshale, S. \& Johnson, E. (2011). Age-related positivity effects and autobiographical memory detail: Evidence from a past/ future source memory task. Memory, 19, 641-652. doi:10.1080/09658211.2011.595723

Jahoda, M. (1958). Current concepts of positive mental health. New York: Basic Books.

Keyes, C., Shmotkin, D. \& Ryff, C. (2002). Optimizing well-being: The empirical encounter of two traditions. Journal of Personality and Social Psychology, 82, 1007-1022. doi:10.1037/0022-3514.82.6.1007

Lobo, A., Saz, P., y Marcos G. (2002). Adaptación del Examen Cognoscitivo Mini-Metal. Madrid: Tea ediciones.

Mather, M. \& Carstensen, L. (2003). Aging and attentional biases for emotional faces. Psychological Science, 14(6), 409-415. doi:10.1111/1467-9280.01455
Mayordomo, T., Sales, A., Satorres, E. \& Meléndez, J. (2016). Bienestar psicológico en función de la etapa de vida, el sexo y su interacción. Pensamiento Psicológico, 14(2), 101-112. doi:10.11144/Javerianacali. PPSI14-2.bpfe

Meléndez, J., Tomás, J. \& Navarro, E. (2007). Análisis de las redes sociales en la vejez a través de la entrevista Manhein de apoyo social. Salud Pública de México, 49(6), 408-416. Recuperado de http://www.redalyc.org/pdf/106/10649607.pdf

Meléndez, J., Tomás, J. \& Navarro, E. (2008). Análisis del bienestar en la vejez según la edad. Revista Española de Geriatría y Gerontología, 43(2), 90-95. doi:10.1016/ S0211-139X(08)71161-2

Meléndez, J., Tomás, J. \& Navarro, E. (2011). Actividades de la vida diaria y bienestar y su relación con la edad y el género en la vejez. Anales de Psicología, 27(1), 164-169. Recuperado de http://www.redalyc.org/ pdf/167/16717018019.pdf

Morganti, J., Nehrke, M., Hulicka, I. \& Cataldo, J. (1988). Life-span differences in life satisfaction, self-concept, and locus of control. International Journal of Aging and Human Development, 26(1), 45-56. doi:10.2190/HDAD-XT0C-W8JB-63DR

Mroczek, D. \& Kolarz, C. (1998). The effect of age on positive and negative affect: A developmental perspective on happiness. Journal of Personality and Social Psychology, 75, 1333-1349. doi:10.1037/00223514.75.5.1333

Neugarten, B. (1973). Personality change in late life: A developmental perspective. En C. Eisodorfer \& M. Lawton (Eds.), The psychology of adult development and aging (pp. 311-335). Washington, DC: American Psychological Association.

Nolla, M., Queral, R. \& Miró, J. (2014). Las escalas PANAS de afecto positivo y negativo: nuevos datos de su uso en personas mayores. Revista de Psicopatología y Psicología Clínica, 19(1), 15-21. Recuperado de http://revistas.uned.es/index.php/RPPC/article/ view/12931/pdf_32

Okun, M. \& Stock, W. (1987). Correlates and components of subjective wellbeing among the elderly. Journal of Applied Gerontology, 6, 95-112. doi:10.1177/073346488700600108

Pasupathi, M. \& Carstensen, L. (2003). Age and emotional experience during mutual reminiscing. Psychology \& Aging, 18, 430-442. doi:10.1037/0882-7974.18.3.430

Ryan, R. \& Deci, E. (2001). On happiness and human potentials: A review of research on hedonic and eudaimonic well-being. Annual Review of Psychology, 52, 141-166. doi:10.1146/annurev.psych.52.1.141

Ryff, C. (1989). Happiness is everything: or is it? Explorations on the meaning of psychological well-being. Journal of Personality and Social Psychology, 57, 10691081. doi:10.1037/0022-3514.57.6.1069

Ryff, C. (1991). Possible selves in adulthood and old age: A tale of shifting horizons. Psychology and Aging, 6, 286295. doi:10.1037/0882-7974.6.2.286

Ryff, C. (2002). Optimizing well-being: The empirical encounter of two traditions. Journal of Personality and Social Psychology, 82, 1007-1022. doi:10.1037/00223514.82.6.1007

Ryff, C. (2014). Psychological well-being revisited: Advances in the science and practice of eudaimonia. 
Psychotherapy and Psychosomatics, 83, 10-28. doi:10.1159/000353263

Ryff, C. \& Keyes, C. (1995). The Structure of psychological well-being revisited. Journal of Personality and Social Psychology, 69, 719-727. doi:10.1037/00223514.69.4.719

Sandín, B., Chorot, P., Lostao, L., Joiner, T., Santed, M. \& Valiente, R. (1999). Escalas PANAS de afecto positivo y negativo: Validación factorial y convergencia transcultural. Psicothema, 11(1), 37-51. Recuperado de http://www.psicothema.com/pdf/229.pdf

Schryer, E. \& Ross, M. (2014). Does the age-related positivity effect in autobiographical recall reflect differences in appraisal or memory? The Journals of Gerontology Series B: Psychological Sciences and Social Sciences, 69(4), 548-556. doi:10.1093/geronb/ gbt047

Seligman, M. \& Csikszentmihalyi, M. (2000). Positive psychology: An introduction. American Psychologist, 55(1), 5-14. doi:10.1037//0003-066X.55.1.5

Sims, T., Hogan, C. \& Carstensen, L. (2015). Selectivity as an emotion regulation strategy: Lessons from older adults. Current Opinion in Psychology, 3, 80-84. doi:10.1016/j.copsyc.2015.02.012

Veenhoven, R. (1994). El estudio de la satisfacción con la vida. Intervención Psicosocial, 3, 87-116. Recuperado de https://repub.eur.nl/pub/16195

Watson, D., Clark, L. \& Tellegen, A. (1988). Development and validation of brief measures of positive and negative affect: the PANAS scales. Journal of Personality and Social Psychology, 54(6), 1063-1070. doi:10.1037/0022-3514.54.6.1063 